\title{
Deformation-based droplet separation in microfluidics
}

\author{
Yunju Chang', Xinye Chen ${ }^{2}$, Yuting Zhou³, Jiandi Wan*3
}

1 : Department of Materials Science and Engineering, University of California, Davis, Davis, CA 95616, United States

2 : Microsystems Engineering, Rochester Institute of Technology, Rochester, NY 14623, United States

3 : Department of Chemical Engineering, University of California, Davis, Davis, CA 95616, United States

\section{Corresponding Author (*)}

Email address : jdwan@ucdavis.edu 


\section{SUPPORTING INFORMATION.}

\begin{tabular}{|c|c|c|c|c|c|c|c|}
\hline $\begin{array}{c}\text { Glycerol } \\
(w \mathrm{t} \%)\end{array}$ & $\begin{array}{c}\text { Size } \\
D_{d}(\mu \mathrm{m})\end{array}$ & $\begin{array}{l}\text { Velocity } \\
D_{v}(\mathrm{~m} / \mathrm{s})\end{array}$ & $\begin{array}{c}\text { Surface } \\
\text { tension } \\
\sigma\left(\mathbf{m N} \cdot \mathbf{m}^{-1}\right)\end{array}$ & $\begin{array}{c}\text { Viscosity } \\
D_{\mu}(\mathbf{m P a} \cdot \mathbf{s})\end{array}$ & $\begin{array}{c}\text { Density } \\
\rho\left(\mathbf{k g} / \mathbf{m}^{3}\right)\end{array}$ & $\boldsymbol{R e}$ & $C a$ \\
\hline 25 & \multirow{6}{*}{26} & \multirow{6}{*}{0.02} & \multirow{6}{*}{35.52} & 2.1 & 1061 & 0.263 & 0.0012 \\
\hline 45 & & & & 5.7 & 1111 & 0.101 & 0.0032 \\
\hline 62 & & & & 9.7 & 1146 & 0.061 & 0.0055 \\
\hline 80 & & & & 49.3 & 1182 & 0.012 & 0.0278 \\
\hline 85 & & & & 84.4 & 1204 & 0.007 & 0.0475 \\
\hline 90 & & & & 159.8 & 1236 & 0.004 & 0.0900 \\
\hline
\end{tabular}

\begin{tabular}{|c|c|c|c|c|c|}
\hline \multirow{2}{*}{$\begin{array}{c}\text { Glycerol } \\
(\mathbf{w t} \%)\end{array}$} & $\begin{array}{c}\text { Velocity } \\
\boldsymbol{D}_{\boldsymbol{v}}(\mathbf{m} / \mathbf{s})\end{array}$ & $\boldsymbol{C} \boldsymbol{a}$ & \multicolumn{3}{|c|}{$\boldsymbol{R} \boldsymbol{e}$} \\
\cline { 2 - 6 } & 0.02 & 0.0012 & $\boldsymbol{D}_{\boldsymbol{d}}=\mathbf{2 6} \boldsymbol{\mu \mathbf { m }}$ & $\boldsymbol{D}_{\boldsymbol{d}}=\mathbf{3 2} \boldsymbol{\mu \mathbf { m }}$ & $\boldsymbol{D}_{\boldsymbol{d}}=\mathbf{4 0} \boldsymbol{\mu \mathbf { m }}$ \\
\hline \multirow{4}{*}{$\mathbf{2 5}$} & 0.03 & 0.0018 & 0.263 & 0.323 & 0.404 \\
\cline { 2 - 6 } & 0.04 & 0.0024 & 0.525 & 0.485 & 0.606 \\
\cline { 2 - 6 } & 0.05 & 0.0030 & 0.657 & 0.808 & 0.808 \\
\hline \multirow{4}{*}{$\mathbf{4 5}$} & 0.02 & 0.0032 & 0.101 & 0.125 & 0.156 \\
\cline { 2 - 6 } & 0.03 & 0.0048 & 0.152 & 0.187 & 0.234 \\
\cline { 2 - 6 } & 0.04 & 0.0064 & 0.203 & 0.249 & 0.312 \\
\cline { 2 - 6 } & 0.05 & 0.0080 & 0.253 & 0.312 & 0.390 \\
\hline \multirow{4}{*}{$\mathbf{6 2}$} & 0.02 & 0.0055 & 0.061 & 0.076 & 0.095 \\
\cline { 2 - 6 } & 0.03 & 0.0082 & 0.092 & 0.113 & 0.142 \\
\cline { 2 - 6 } & 0.04 & 0.0109 & 0.123 & 0.151 & 0.189 \\
\cline { 2 - 6 } & 0.05 & 0.0137 & 0.154 & 0.189 & 0.236 \\
\hline \multirow{4}{*}{$\mathbf{8 0}$} & 0.02 & 0.0278 & 0.012 & 0.015 & 0.019 \\
\cline { 2 - 6 } & 0.03 & 0.0416 & 0.019 & 0.023 & 0.029 \\
\cline { 2 - 6 } & 0.04 & 0.0555 & 0.025 & 0.031 & 0.038 \\
\cline { 2 - 6 } & 0.05 & 0.0694 & 0.031 & 0.038 & 0.048 \\
\hline \multirow{4}{*}{$\mathbf{8 5}$} & 0.02 & 0.0475 & 0.007 & 0.009 & 0.011 \\
\cline { 2 - 6 } & 0.03 & 0.0713 & 0.011 & 0.014 & 0.017 \\
\cline { 2 - 6 } & 0.04 & 0.0950 & 0.015 & 0.018 & 0.023 \\
\cline { 2 - 6 } & 0.05 & 0.1188 & 0.019 & 0.023 & 0.029 \\
\hline \multirow{4}{*}{$\mathbf{9 0}$} & 0.02 & 0.0900 & 0.004 & 0.005 & 0.006 \\
\cline { 2 - 6 } & 0.03 & 0.1350 & 0.006 & 0.007 & 0.009 \\
\cline { 2 - 6 } & 0.04 & 0.1800 & 0.008 & 0.010 & 0.012 \\
\cline { 2 - 6 } & 0.05 & 0.2249 & 0.010 & 0.012 & 0.015 \\
\hline
\end{tabular}

Table S1. Calculated Reynolds number and capillary number of droplets. 
A

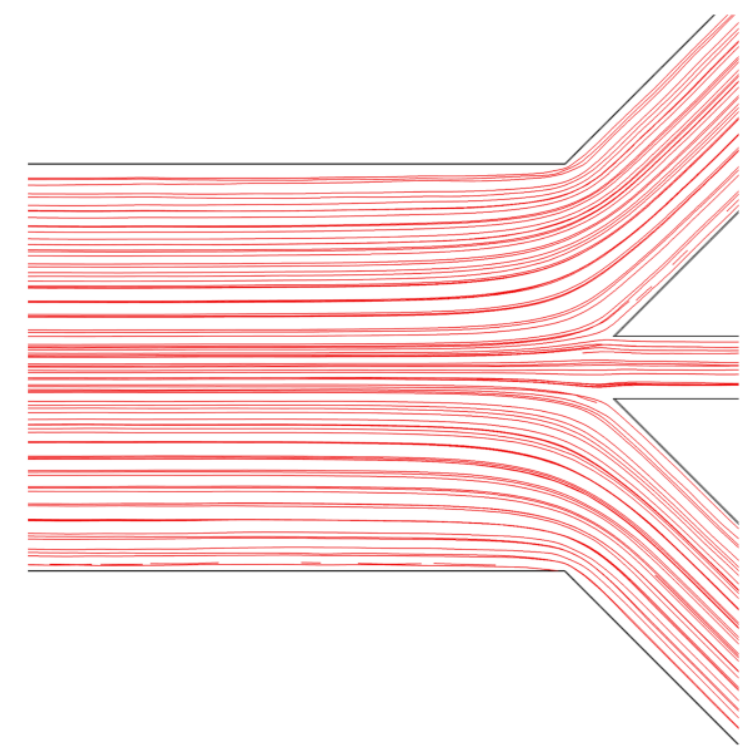

B

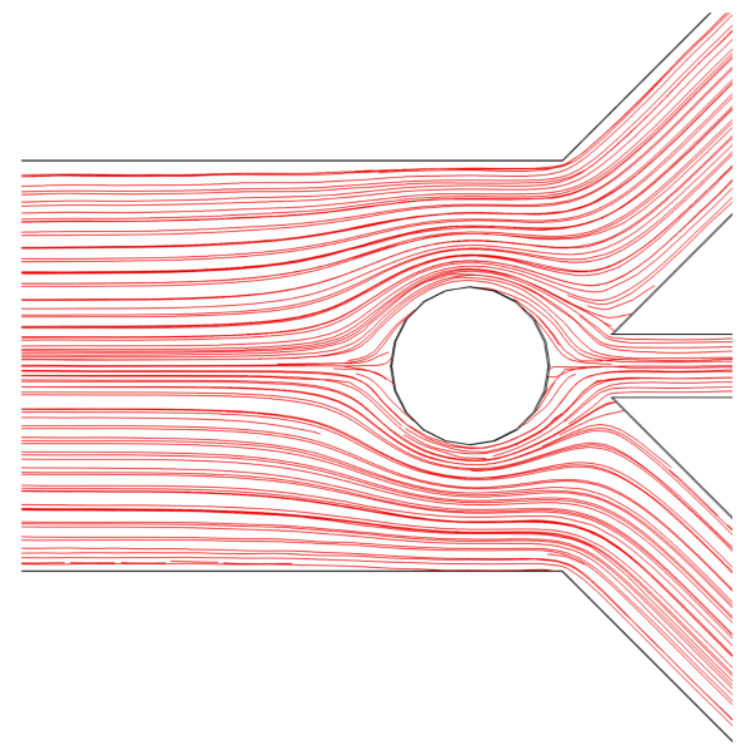

Figure S1. Simulation of fluid streamline inside microfluidic sorting device. (A). Fluid streamline without post. (B). Fluid streamline with a post. The gap distance is $40 \mu \mathrm{m}$. 


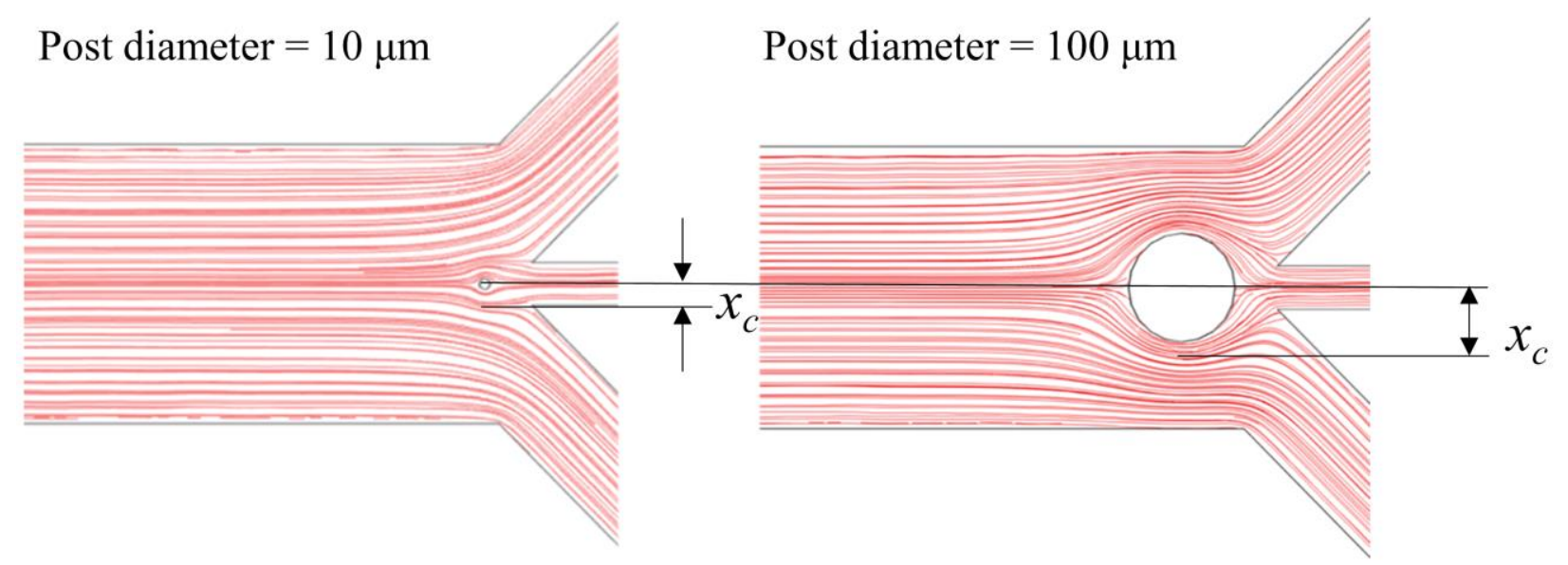

$x_{c}=\operatorname{critical} x$ below which all streamline exit to the center outlet

Figure S2. The critical $x, x_{c}$, changes by changing the diameter of post. (A). Simulation of fluid streamline showing $x_{c}$ with a post of $10 \mu \mathrm{m}$ and (B) $100 \mu \mathrm{m}$ diameter. 
A

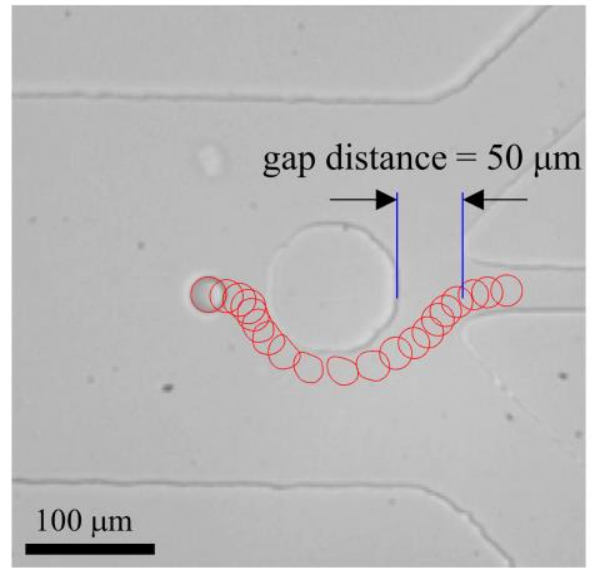

B

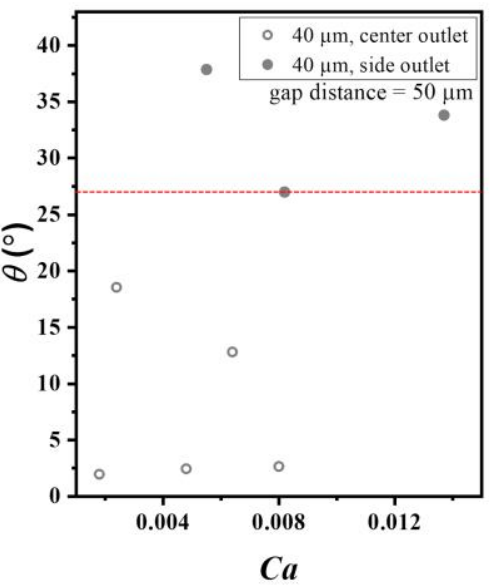

C

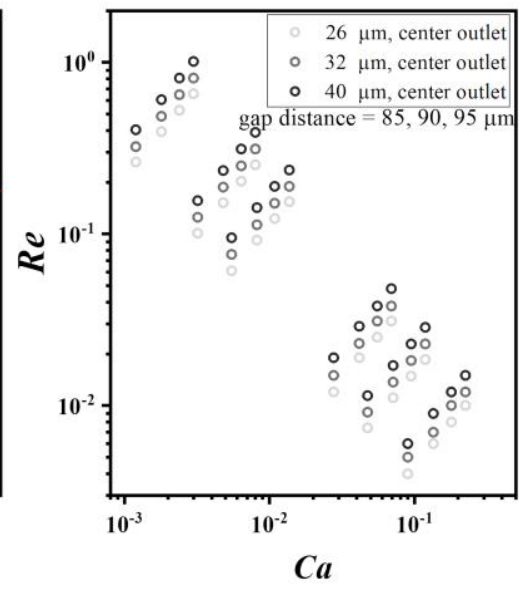

Figure S3. Droplet separation with $50 \mu \mathrm{m}$ gap distance only occurred for droplet with a diameter of $40 \mu \mathrm{m}$. (A). Representative image of droplet trajectory for 26 and $32 \mu \mathrm{m}$ size droplets. There was no impact on the central sorting channel. (B). Droplets with $D_{d}=40 \mu \mathrm{m}$ were sorted to the side outlets when the impact angle was higher than $27^{\circ}$. (C). As the gap distance was further increased to 85,90 , and $95 \mu \mathrm{m}$, droplet separation to the side outlets did not occur regardless of droplet size, viscosity, or velocity. 